\title{
Acute progression of untreated incidental WHO Grade II glioma to glioblastoma in an asymptomatic patient
}

\author{
Jérôme Cochereau, ${ }^{1}$ Guillaume Herbet, $\mathrm{PhD},{ }^{1,2}$ Valérie Rigau, MD, $\mathrm{PhD},{ }^{2,3}$ and \\ Hugues Duffau, MD, PhD',2
}

\begin{abstract}
${ }^{1}$ Department of Neurosurgery, Gui de Chauliac Hospital, Montpellier University Medical Center; ${ }^{2}$ Institute for Neuroscience of Montpellier, INSERM U1051, Team "Plasticity of Central Nervous System, Human Stem Cells and Glial Tumors," Saint Eloi Hospital, Montpellier University Medical Center; and 'Tumor Cellular and Tissular Biopathology Department, Gui de Chauliac Hospital, Montpellier University Medical Center, Montpellier, France
\end{abstract}

\begin{abstract}
WHO Grade II glioma (low-grade glioma [LGG]) is increasingly diagnosed as an incidental finding in patients undergoing MRI for many conditions. Recent data have demonstrated that such incidental LGGs are progressive tumors that undergo clinical transformation and ultimately become malignant. Although asymptomatic LGG seems to represent an earlier step in the natural course of a glioma than the symptomatic LGG, it is nonetheless impossible to predict at the individual level when the tumor will become malignant. The authors report the case of a 43-year-old woman with a right operculo-insular LGG that was incidentally diagnosed because of headaches. No treatment was proposed, and repeated MRI scans were performed for 6 years in another institution. Due to a slow but continuous growth of the lesion, the patient was finally referred to our center to undergo surgery. Interestingly, objective calculation of the velocity of the tumor's diametric expansion demonstrated a sudden acceleration of the growth rate within the 5 months preceding surgery, with the development of contrast enhancement. Remarkably, the patient was still asymptomatic. An awake resection was performed with intraoperative electrical mapping. There was no functional worsening following surgery, as assessed on postoperative neuropsychological examination. Removal of $92 \%$ of signal abnormality on FLAIR MRI was achieved, with complete resection of the area of contrast enhancement. Neuropathological examination revealed a glioblastoma, and the patient was subsequently treated with concomitant radiotherapy and chemotherapy. Although a "wait and see" attitude has been advocated by some authors with respect to incidental LGG, our original case demonstrates that acute transformation to glioblastoma may nonetheless occur, even before the onset of any symptoms. Therefore, because the lack of symptoms does not protect from malignant transformation, we propose consideration of earlier resection in a more systematic manner in cases of incidental LGG.
\end{abstract}

http://thejns.org/doi/abs/10.3171/2014.12.JNS141851

KEY WORDS low-grade glioma; incidental tumor; malignant transformation; surgery; oncology

$\mathrm{W}$ ORLD Health Organization (WHO) Grade II glioma (diffuse low-grade glioma [LGG]) is a continuously growing lesion that migrates along white matter pathways and will invariably evolve toward a higher grade of malignancy. ${ }^{4}$ Despite the lack of Class I evidence, a growing amount of data in the modern literature supports a significant impact of maximal resection on overall survival. ${ }^{1,10,13,20,23}$ Therefore, surgery is currently the first therapeutic option to consider in LGG. ${ }^{8}$ Interestingly, it was demonstrated that the natural history of incidental LGG (iLGG) was similar to the spontaneous course of symptomatic LGG, that is, with a constant growth and risk of malignant transformation..$^{16}$ This is the reason why surgery has recently been proposed for iLGG. ${ }^{5}$ Indeed, preliminary reports showed that the extent of resection (EOR) was higher in iLGG than in symptomatic LGG, due to smaller tumor volume, ${ }^{19}$ and that it was possible to preserve the patients' quality of life thanks to the use of awake mapping. ${ }^{3}$ Nonetheless, the timing of surgery is not well defined in iLGG, because it is impossible to predict at the individual level when malignant transformation will occur. One could expect that the risk of degeneration is

ABBREVIATIONS EOR = extent of resection; FLAIR = fluid attenuation inversion recovery; iLGG = incidental LGG; LGG = low-grade glioma; WHO = World Health Organization.

SUBMITTED August 10, 2014. ACCEPTED December 12, 2014

INCLUDE WHEN CITING Published online July 10, 2015; DOI: 10.3171/2014.12.JNS141851.

DISCLOSURE The authors report no conflict of interest concerning the materials or methods used in this study or the findings specified in this paper. 
basically nil before the onset of any symptoms (usually seizures), explaining why some authors have suggested a "wait and see attitude" in iLGGs. ${ }^{21,22}$

Here, we report, for the first time to our knowledge, a case demonstrating the acute radiological and neuropathological transformation of iLGG to a glioblastoma in an untreated patient who remained free of symptoms.

\section{Case Report}

\section{History and Examination}

This 43-year-old left-handed woman, an architect with no previous significant medical history, underwent brain MRI examination in May 2008 because of headaches. This MRI study revealed a small left carotido-ophthalmic aneurysm (untreated) as well as a right insular lesion highly suggestive of an LGG (nonenhancing hypointense lesion on T1-weighted MRI, hyperintense on fluid attenuation inversion recovery [FLAIR] MRI) (Fig. 1 left). No definitive histological diagnosis was obtained at the time of the initial finding. Close clinical and radiological follow-up was performed over the next 6 years at another institution, including MRI studies every 12 months. The patient enjoyed a normal familial, social, and professional life, with no symptoms (of particular significance, no seizures) and no treatment (no antiepileptic drugs). Due to the lesion's slow but continuous growth, the patient was finally referred to our center in February 2014 to consider possible surgery. At that time, we retrospectively calculated the volumes on the first and last MRI studies using dedicated software (Myrian, Intrasense). On the first imaging study (May 2008), the lesion volume was $3 \mathrm{~cm}^{3}$ (Fig. 1 left), whereas on the last imaging study (November 2013), it was $11 \mathrm{~cm}^{3}$ (Fig. 1 right). Therefore, the mean spontaneous velocity of diametric expansion before firstline treatment, i.e., the slope of the mean tumor diameter growth curve deduced using the methodology detailed by Pallud et al., was $1.8 \mathrm{~mm} /$ year. ${ }^{18}$ This is in agreement with a natural course of LGG. ${ }^{12}$ At the end of the interview with
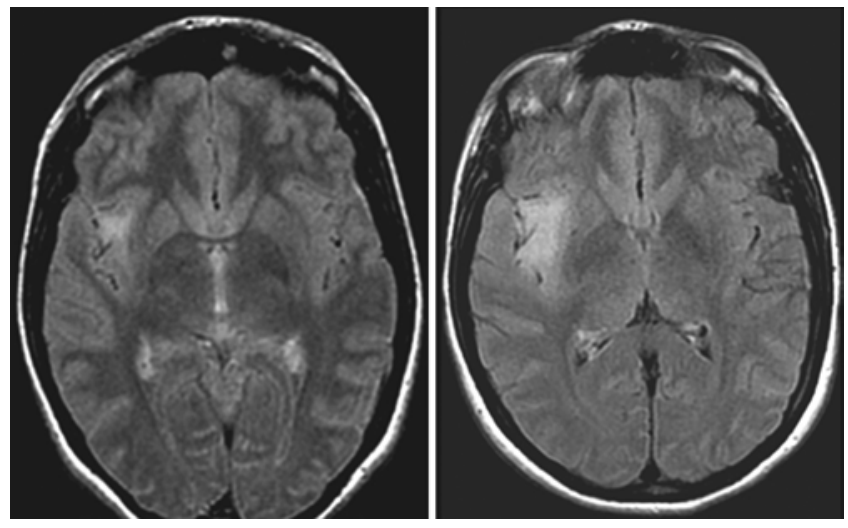

FIG. 1. Left: Axial FLAIR MR image obtained in May 2008 showing a right insular diffuse LGG incidentally discovered because of headache in a 43-year-old left-handed woman with normal findings on neurological examination and enjoying a normal life. The tumor volume was 3 $\mathrm{cm}^{3}$. Right: Axial FLAIR MR image obtained in November 2013 showing a slow but objectively demonstrable tumor growth in a still asymptomatic patient. The volume was $11 \mathrm{~cm}^{3}$. the patient and her family, due to this objective increase of tumor volume over years, surgery was decided upon and planned for April 2014.

\section{Preoperative Assessment}

The results of the preoperative neurological examination were normal. In addition, the neurocognitive assessment performed by a trained neuropsychologist (G.H.) revealed no cognitive impairment in various domains such as executive functioning, social (mentalizing) and spatial cognition, praxis and visuo-perceptive abilities, language, and working memory. However, an MRI study performed on the day before surgery showed an rapid increase in tumor volume (to a volume of $21 \mathrm{~cm}^{3}$ ), indicating an acute acceleration of the growth rate-that is, a growth rate of about $13 \mathrm{~mm} /$ year, in contrast to the previous $1.8 \mathrm{~mm} /$ year-in agreement with the natural history of a highgrade glioma..$^{14,17}$ At this time, the tumor also involved the right frontal operculum. In addition, this imaging study revealed the sudden occurrence of a "ring-like" contrast enhancement in the anterolateral part of the lentiform nucleus, supporting the likelihood of malignant transformation (Fig. 2).

\section{Operation}

This left-handed patient was surgically treated under local anesthesia (asleep-awake-asleep protocol using propofol and remifentanil). A frontotemporal craniotomy was performed, and intraoperative ultrasonography was used to delineate the lesion. A bipolar electrode with 5-mmspaced tips delivering a biphasic current (pulse frequency $60 \mathrm{~Hz}$, single pulse phase duration $1 \mathrm{msec}$, amplitude 2 $\mathrm{mA}$; Nimbus, Hemodia) was applied to the patient's brain to map the cortex before resection. ${ }^{7,26}$ According to a method we previously described, ${ }^{9}$ we instructed the patient to perform a double task associating continuous movement of the left upper limb and picture naming; the aim was to check in real time whether any sensorimotor or speech articulation disorders occurred during the mapping and the resection. A neuropsychologist (G.H.) was present in the operating room to evaluate any functional disturbances. Four eloquent sites were identified at the cortical surface:
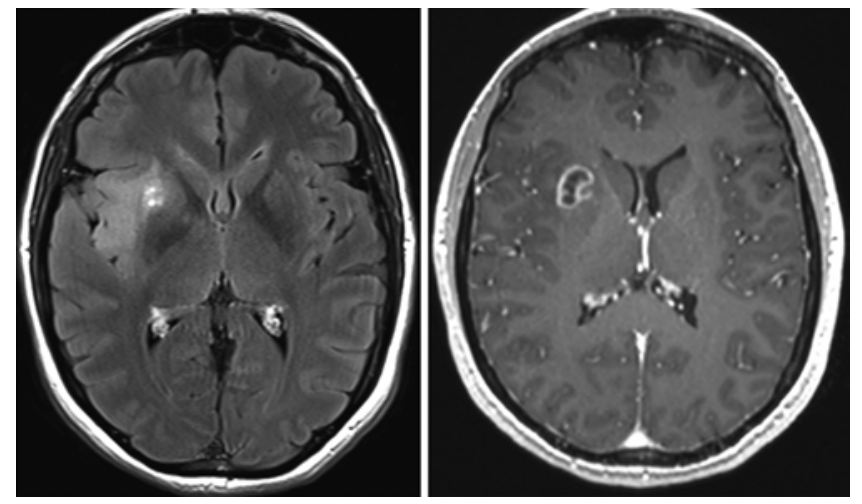

FIG. 2. Immediate preoperative axial FLAIR (left) and axial enhanced T1-weighted (right) MR images (April 2014) showing a rapid increase of the tumor volume $\left(21 \mathrm{~cm}^{3}\right)$ with the sudden occurrence of a "ringlike" contrast enhancement within the anterolateral part of the lentiform nucleus. 
stimulation over the rolandic operculum (ventral premotor cortex) induced anarthria, while more medially over the precentral gyrus, stimulation induced involuntary facial movement (primary motor cortex of the face) and arrest of upper limb movement as well as speech (negative motor area) (Fig. 3a).

The frontal operculum, partly invaded by the tumor, was removed according to these functional boundaries. Thus, the insula was approached through the inferior frontal gyrus using a subpial dissection, and the insular portion of the glioma was resected. During this second surgical stage, resection and subcortical stimulation were used in an alternating way to prevent damage to eloquent deep structures. ${ }^{6,7}$ The resection was extended up to the sites at which stimulation elicited speech arrest, the corona radiata (as the superior and deep limit) and the anterior limb of the internal capsule. The lateral part of the lentiform nucleus (invaded by the enhancing part of the tumor) was also encountered in the depth (Fig. 3b).

\section{Postoperative Course}

The postoperative course was uneventful, with no neurological worsening. The patient had no local or general complications, and she had no seizures. She reached her preoperative neuropsychological baseline only 3 days after surgery and returned home 4 days after surgery.

Postsurgical MRI revealed subtotal resection, with a 92\% extent of resection of the FLAIR signal abnormality, given some residual tumor $\left(1.6 \mathrm{~cm}^{3}\right)$ within the posterior part of the insula. The area of enhancement was totally removed (Fig. 4).

The neuropathological diagnosis was glioblastoma (WHO Grade IV). The molecular analysis revealed IDHI mutation, MGMT methylation, and 1p19 co-deletion.

The patient was subsequently treated with concomitant radiotherapy and chemotherapy (Stupp protocol). ${ }^{25}$
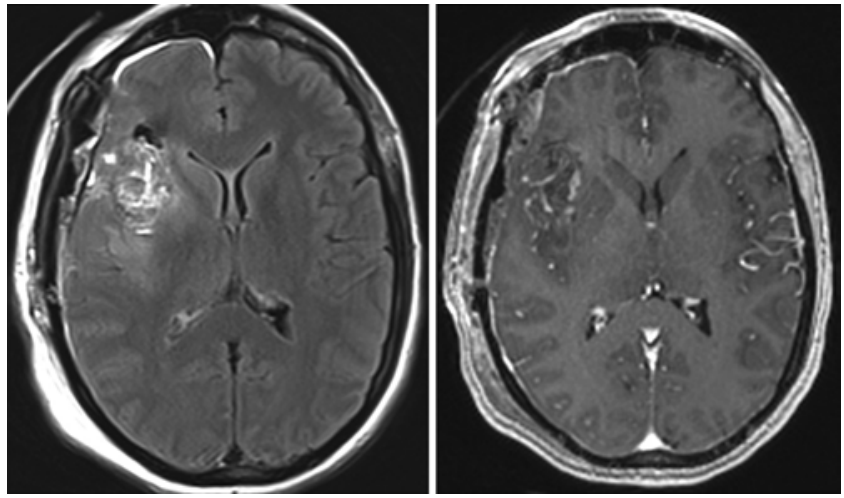

FIG. 4. Immediate postoperative axial FLAIR (left) and axial enhanced T1-weighted (right) MR images revealing a subtotal resection, with a residue $\left(1.6 \mathrm{~cm}^{3}\right)$ within the posterior part of the insula. The area of enhancement was totally removed.

\section{Discussion}

Although early and maximal resection is at present the first-line treatment for symptomatic LGG, as recommended by the current European Guidelines, ${ }^{24}$ management of iLGG is still a matter of debate. This controversy is due, in particular, to a poor knowledge of the natural history of this rare disease. Interestingly, the spontaneous course of iLGG has recently been investigated, showing that this entity is a progressive tumor in all cases, with a median velocity of diametric expansion of approximately $3.5 \mathrm{~mm} /$ year (that is, very close to the growth rate of symptomatic LGG) and leading to clinical transformation toward symptomatic LGG at a median interval of 48 months after radiological discovery. ${ }^{16} \mathrm{~A}$ similar growth rate was confirmed by Potts et al.,19 based upon an experience with 35 iLGGs. These data demonstrate that iLGG is never stable. Above all, in the series by Pallud et al., ${ }^{16}$ almost one-third
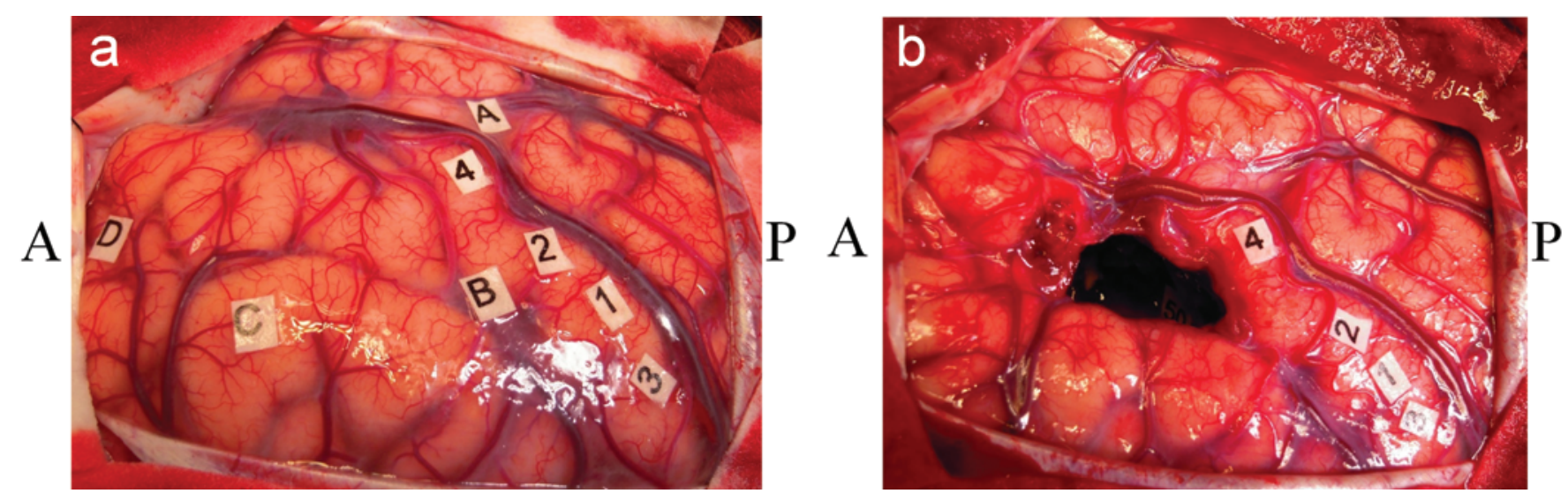

FIG. 3. a: Intraoperative photograph obtained during awake surgery. After the lesion boundaries (letter tags) were delineated by means of ultrasonography, 4 eloquent sites were identified at the cortical surface with electrical mapping (number tags). Stimulation over the rolandic operculum (ventral premotor cortex) induced anarthria (Tag 4). More medially over the precentral gyrus, stimulation induced involuntary facial movement (primary motor cortex of the face) (Tags 1 and 2) and arrest of upper-limb movement as well as speech (negative motor area) (Tag 3). b: View after glioma removal, achieved according to functional boundaries detected by intraoperative electrical mapping both at cortical and subcortical levels. Indeed, the resection was extended up to the corona radiata (as the superior and deep limit) and the anterior limb of the internal capsule, which both elicited speech arrest when stimulated. The lateral part of the lentiform nucleus was also encountered in the depth. There were no intraoperative seizures. $A=$ anterior; $\mathrm{P}=$ posterior. Figure is available in color online only. 
of cases (14 of 47) demonstrated malignant transformation at a median interval of 5.7 years after radiological discovery, and 4 patients died at a median interval of 8.9 years after radiological discovery of the lesion. In the same vein, in a surgical series with $11 \mathrm{iLGGs,} \mathrm{a} \mathrm{microfocus} \mathrm{of} \mathrm{ana-}$ plasia with endothelial proliferation was identified in the middle of the tumor in $27 \%$ of cases, demonstrating that malignant transformation may occur before any symptoms. ${ }^{3}$

Here, we report, for the first time to our knowledge, a case of an iLGG that progressed to glioblastoma in an asymptomatic patient. While it is well known that about $10 \%$ of glioblastomas are derived from WHO Grade II tumors, it is very uncommon to observe the acute and direct transformation of an LGG into a glioblastoma without any intervening progression to WHO Grade III glioma (the so-called "anaplastic transformation"). To our knowledge, this phenomenon has never been documented in real time in the literature by serial MRI. In addition, it is exceptional to show that this progression to glioblastoma can occur before the onset of symptoms: this is the first such case ever reported. Although there are no reliable criteria to predict at the individual level when malignant transformation will occur in iLGG, one could hypothesize that the risk of degeneration is basically nil before the onset of any symptoms. This is the reason why a conservative attitude has been proposed by authors who recommended repeated MRIs..$^{21,22}$ This is exactly what was done in the present case in a previous institution - that is, a wait and watch approach. Nonetheless, this case taught us that the lack of symptoms does not protect from malignant transformation. Indeed, even in the postoperative period, after malignant transformation was histologically confirmed, our patient did not experience seizures, neurological deficits, or neurocognitive disturbances, as determined by comparison with the preoperative neuropsychological examination. In addition, this case demonstrates that a real iLGG, i.e., a lesion followed over years with a slow growth rate of approximately $1.8 \mathrm{~mm} / \mathrm{year}$, may nonetheless progress acutely to glioblastoma without passing through the step of WHO Grade III glioma.

Even though we cannot claim that earlier surgery would have prevented degeneration in this case, we can nonetheless assume that earlier surgery would have provided a greater extent of resection (EOR). Indeed, it has already been shown that the EOR is higher in iLGG than in symptomatic LGG, due to smaller tumor volumes. ${ }^{16,19}$ In a previous surgical experience with 11 iLGGs, beyond the fact there was no partial resection, a total resection was achieved in $36 \%$ of cases and a supratotal resection (i.e., the resection of a margin around the tumor visible on FLAIR MRI) was achieved in $27 \%$ of cases. ${ }^{3}$ This issue is a crucial one because a supratotal removal may prevent malignant transformation in $\mathrm{LGG}^{27}$ In the same vein, several authors demonstrated that patients operated on for iLGG had a significant improvement in overall survival in comparison with a control group of patients with symptomatic LGG. ${ }^{16,19}$ As a consequence, taken together, these data plead in favor of an early surgical treatment in asymptomatic patients, to maximize the EOR before malignant transformation.
Finally, from a functional point of view, one could suggest that early resection is not appropriate for iLGG, due to the surgical risk. However, it has been shown that maximal resection of iLGG is possible with no permanent postoperative deficit, even when eloquent areas are involved, ${ }^{3}$ thanks to the use of intraoperative mapping in awake patients. In the present case, direct stimulation enabled the achievement of a functional mapping-guided resection, both at the cortical level (identification of the primary motor cortex of the face, ventral premotor cortex, and negative motor area) and at the subcortical level (detection of the fibers running in the corona radiata and anterior limb of the internal capsule, involved in speech). Functional preservation was confirmed by the immediate postoperative neuropsychological examination, which revealed no neurological or cognitive deficits. Therefore, it seems that the theoretical risk of generating neurological worsening does not represent an argument against early surgery in iLGG.

In summary, we are recommending early surgery for patients with any incidentally found lesion resembling a LGG, after the demonstration of tumor growth-which strongly suggests that the lesion is a glioma. ${ }^{16}$ Indeed, in a recent prospective series of 21 iLGG patients who underwent surgery with a mean follow-up of 49 months following resection, all patients are still alive and enjoy a normal life, with no immediate postoperative seizures in any case but one. ${ }^{2}$ These data strongly reinforce the proposal of a screening policy for LGG, to evolve toward a "preventive treatment." 11,15

\section{Conclusions}

With this original case, we highlight the fact that an iLGG can undergo acute and direct transformation into glioblastoma, even in a patient who remains asymptomatic. Because the lack of symptoms does not protect from malignant transformation, we propose that earlier resection be considered in a more systematic manner in cases of iLGG, with the aim of increasing the EOR while preserving the patients' quality of life.

\section{References}

1. Capelle L, Fontaine D, Mandonnet E, Taillandier L, Golmard JL, Bauchet L, et al: Spontaneous and therapeutic prognostic factors in adult hemispheric World Health Organization Grade II gliomas: a series of 1097 cases: clinical article. J Neurosurg 118:1157-1168, 2013

2. de Oliveira Lima GL, Duffau H: Is there a risk of seizures in "preventive" awake surgery for incidental diffuse low-grade gliomas? J Neurosurg [epub ahead of print February 27, 2015. DOI: 10.3171/2014.9.JNS141396]

3. Duffau H: Awake surgery for incidental WHO grade II gliomas involving eloquent areas. Acta Neurochir (Wien) 154:575-584, 2012

4. Duffau H (ed): Diffuse Low-Grade Gliomas in Adults: Natural History, Interaction with the Brain, and New Individualized Therapeutic Strategies. London: Springer, 2013

5. Duffau H: The rationale to perform early resection in incidental diffuse low-grade glioma: toward a "preventive surgical neurooncology.” World Neurosurg 80:e115-e117, 2013

6. Duffau H, Gatignol P, Mandonnet E, Peruzzi P, Tzourio- 
Mazoyer N, Capelle L: New insights into the anatomofunctional connectivity of the semantic system: a study using cortico-subcortical electrostimulations. Brain 128:797-810, 2005

7. Duffau H, Peggy Gatignol ST, Mandonnet E, Capelle L, Taillandier L: Intraoperative subcortical stimulation mapping of language pathways in a consecutive series of 115 patients with Grade II glioma in the left dominant hemisphere. J Neurosurg 109:461-471, 2008

8. Duffau H, Taillandier L: New concepts in the management of diffuse low-grade glioma: Proposal of a multistage and individualized therapeutic approach. Neuro Oncol 17:332-342, 2015

9. Fernández Coello A, Moritz-Gasser S, Martino J, Martinoni M, Matsuda R, Duffau H: Selection of intraoperative tasks for awake mapping based on relationships between tumor location and functional networks. J Neurosurg 119:13801394,2013

10. Jakola AS, Myrmel KS, Kloster R, Torp SH, Lindal S, Unsgård G, et al: Comparison of a strategy favoring early surgical resection vs a strategy favoring watchful waiting in lowgrade gliomas. JAMA 308:1881-1888, 2012

11. Mandonnet E, de Witt Hamer P, Pallud J, Bauchet L, Whittle I, Duffau H: Silent diffuse low-grade glioma: toward screening and preventive treatment? Cancer 120:1758-1762, 2014

12. Mandonnet E, Delattre JY, Tanguy ML, Swanson KR, Carpentier AF, Duffau H, et al: Continuous growth of mean tumor diameter in a subset of grade II gliomas. Ann Neurol 53:524-528, 2003

13. McGirt MJ, Chaichana KL, Attenello FJ, Weingart JD, Than $\mathrm{K}$, Burger PC, et al: Extent of surgical resection is independently associated with survival in patients with hemispheric infiltrating low-grade gliomas. Neurosurgery 63:700-708, 2008

14. Pallud J, Blonski M, Mandonnet E, Audureau E, Fontaine D, Sanai N, et al: Velocity of tumor spontaneous expansion predicts long-term outcomes for diffuse low-grade gliomas. Neuro Oncol 15:595-606, 2013

15. Pallud J, Capelle L, Taillandier L, Badoual M, Duffau H, Mandonnet E: The silent phase of diffuse low-grade gliomas. Is it when we missed the action? Acta Neurochir (Wien) 155:2237-2242, 2013

16. Pallud J, Fontaine D, Duffau H, Mandonnet E, Sanai N, Taillandier L, et al: Natural history of incidental World Health Organization grade II gliomas. Ann Neurol 68:727-733, 2010

17. Pallud J, Mandonnet E, Duffau H, Kujas M, Guillevin R, Galanaud D, et al: Prognostic value of initial magnetic resonance imaging growth rates for World Health Organization grade II gliomas. Ann Neurol 60:380-383, 2006

18. Pallud J, Taillandier L, Capelle L, Fontaine D, Peyre M, Ducray F, et al: Quantitative morphological magnetic resonance imaging follow-up of low-grade glioma: a plea for systematic measurement of growth rates. Neurosurgery 71:729-740, 2012

19. Potts MB, Smith JS, Molinaro AM, Berger MS: Natural history and surgical management of incidentally discovered low-grade gliomas. J Neurosurg 116:365-372, 2012

20. Schomas DA, Laack NN, Rao RD, Meyer FB, Shaw EG, O'Neill BP, et al: Intracranial low-grade gliomas in adults: 30-year experience with long-term follow-up at Mayo Clinic. Neuro Oncol 11:437-445, 2009

21. Shah AH, Madhavan K, Heros D, Raper DM, Iorgulescu JB, Lally BE, et al: The management of incidental low-grade gliomas using magnetic resonance imaging: systematic review and optimal treatment paradigm. Neurosurg Focus 31(6):E12, 2011

22. Shah AH, Madhavan K, Sastry A, Komotar RJ: Managing intracranial incidental findings suggestive of low-grade glioma: learning from experience. World Neurosurg 80:e75-e77, 2013

23. Smith JS, Chang EF, Lamborn KR, Chang SM, Prados MD, Cha S, et al: Role of extent of resection in the long-term outcome of low-grade hemispheric gliomas. J Clin Oncol 26:1338-1345, 2008

24. Soffietti R, Baumert BG, Bello L, von Deimling A, Duffau $H$, Frénay M, et al: Guidelines on management of low-grade gliomas: report of an EFNS-EANO Task Force. Eur J Neurol 17:1124-1133, 2010

25. Stupp R, Mason WP, van den Bent MJ, Weller M, Fisher $\mathrm{B}$, Taphoorn MJ, et al: Radiotherapy plus concomitant and adjuvant temozolomide for glioblastoma. N Engl J Med 352:987-996, 2005

26. Tate MC, Herbet G, Moritz-Gasser S, Tate JE, Duffau H: Probabilistic map of critical functional regions of the human cerebral cortex: Broca's area revisited. Brain 137:27732782, 2014

27. Yordanova YN, Moritz-Gasser S, Duffau H: Awake surgery for WHO Grade II gliomas within "noneloquent" areas in the left dominant hemisphere: toward a "supratotal" resection. Clinical article. J Neurosurg 115:232-239, 2011

\section{Author Contributions}

Conception and design: Duffau, Cochereau. Acquisition of data: all authors. Analysis and interpretation of data: all authors. Drafting the article: Duffau, Cochereau. Critically revising the article: all authors. Reviewed submitted version of manuscript: all authors. Approved the final version of the manuscript on behalf of all authors: Duffau. Study supervision: Duffau.

\section{Correspondence}

Hugues Duffau, Department of Neurosurgery, Gui de Chauliac Hospital, CHU Montpellier, 80 Ave. Augustin Fliche, 34295 Montpellier, France. email: h-duffau@chu-montpellier.fr. 